\title{
Nevus-Associated versus de novo Melanoma: Do They Have Different Characteristics and Prognoses?
}

\author{
Nilay Dumana Gül Erkin ${ }^{b}$ Özay Gököz ${ }^{c}$ Sevilay Karahan ${ }^{d}$ \\ Aycan Uğur Kayıkçıoğlue ${ }^{e}$ İsmail Çelik ${ }^{f}$ \\ a Department of Dermatology, Afyon Kocatepe University, Afyonkarahisar, and ${ }^{b}$ Department \\ of Dermatology, Ankara Guven Hospital, and Departments of ${ }^{\mathrm{C}}$ Pathology, ${ }^{\mathrm{d}}$ Biostatistics, \\ e Plastic and Reconstructive Surgery and fPreventive Oncology, School of Medicine, \\ Hacettepe University, Ankara, Turkey
}

\section{Key Words}

Nevus-associated melanoma $\cdot$ De novo melanoma $\cdot$ Prognosis

\section{Abstract}

Aim: The aim of this study was to determine if nevus-associated melanoma differs in characteristics and prognosis from de novo melanoma. Patients and Methods: The study included 118 melanoma patients. Clinical findings were retrospectively evaluated. For histopathological parameters, HE sections were reexamined. The differentiation between de novo and nevus-associated melanoma was based on the histopathological evidence of a precursor nevus. In addition, all analyses were repeated in all cases in which nevus-associated melanoma was defined based on patient anamnesis. Results: Among all patients, 28 (23.7\%) had nevus-associated melanoma. Nevus-associated melanoma was most commonly located on the extremities (50\%), followed by the trunk (25\%), whereas de novo melanoma was most commonly located in the head and neck region (32.2\%), followed by the acral region (31.1\%). Other clinical findings and histopathological parameters did not differ significantly between the two groups ( $p>0.05$ ). The findings remained consistent following the repeated analysis of all cases in which nevus-associated melanoma was defined based on patient anamnesis. Conclusions: Nevus-associated melanoma was most commonly located on the extremities and the trunk, whereas de novo melanoma was most commonly located in the head and neck and the acral region. Furthermore, nevus-associated melanoma was similar to de novo melanoma in terms of prognosis and other disease characteristics. 


\section{Introduction}

A melanoma is a malignant skin tumor consisting of melanocytes. Although some melanomas arise from preexisting melanocytic nevi, most melanomas develop de novo. Comparative data on melanomas that develop from preexisting melanocytic nevi and de novo are limited, and the effect of the origin of the melanoma on disease characteristics and prognosis remains unclear.

The present study aimed to identify the clinical and histopathological differences between de novo (DNM) and nevus-associated melanoma (NAM) and to determine if prognostic factors and the development of distant metastasis differ according to melanoma origin.

\section{Materials and Methods}

The study included patients diagnosed with melanoma between 2000 and 2010. Clinical data were collected retrospectively from 151 patients, either from their medical records and/ or directly from the patients. For the evaluation of histopathological parameters, formalinfixed, paraffin-embedded, HE-stained sections were reexamined by a dermatologist and a pathologist. Patients with insufficient clinical data and/or histopathological specimens (i.e. punch or shave biopsies) were excluded from the study. The clinical and histopathological data of 118 of the 151 melanoma patients were evaluated and included in this study.

The following clinical parameters were evaluated: age at the time of diagnosis, gender, location of the lesion, lymph node involvement (sentinel or regional), duration of follow-up, development of satellite/in-transit/distant metastasis, and clinical stage. Lesion location was categorized as head and neck region, extremities, trunk, and acral region. The American Joint Committee on Cancer 2009 TNM staging system was used for clinical staging [1].

NAM was diagnosed based on the presence of histopathological evidence of both melanocytic nevus and melanoma in surgically obtained specimens. DNM was defined as melanoma without histopathological evidence of a preexisting nevus. Additional histopathological parameters that were evaluated included lesion diameter, melanoma subtype, Breslow thickness, Clark level, mitotic index, ulceration, regression, lymphocytic infiltration, and lymphovascular/perineural invasion. In each patient, the presence of an associated melanocytic nevus was investigated, and the type of the melanocytic nevus was recorded if determined.

Regression was categorized as partial if it constituted $<75 \%$ of the lesion and as complete if it constituted $\geq 75 \%$ of the lesion. Lymphocytic infiltration was characterized as follows: absent: no lymphocytes present or lymphocytes did not infiltrate the melanoma; non-brisk: focal infiltration; brisk: lymphocytes present throughout the entire vertical growth phase or extending across its entire base [2]. The mitotic index was calculated as the number of mitoses $\left(\mathrm{mm}^{-2}\right)$ and categorized as $<1$ and $\geq 1 \mathrm{~mm}^{-2}$. In addition, for the purpose of comparison, NAM diagnosis was based on patient anamnesis supporting the fact that the presenting lesion had arisen from a preexisting nevus, and all analyses were repeated accordingly. The Hacettepe University School of Medicine Ethics Committee approved the study protocol.

\section{Statistical Methods}

Statistical analysis was performed using SPSS version 15 for Windows (SPSS, Inc., Chicago, Ill., USA). Continuous variables are presented as the mean \pm SD and categorical variables as frequency and percentage. The $\chi^{2}$ test was used to identify associations between categorical variables. Differences in normally distributed variables between groups were analyzed via the independent samples $t$ test, and the Mann-Whitney U test was used for variables not normally distributed. The Kruskal-Wallis test was used to compare $>2$ groups. The level of statistical significance was set at $\mathrm{p}<0.05$. 
Table 1. Demographic and clinical features of the NAM and DNM patients

\begin{tabular}{l|l}
\hline Dermatopathology 2015;2:46-51 \\
\hline DOI: $10.1159 / 000375490$ & $\begin{array}{l}\text { @ 2015 S. Karger AG, Basel } \\
\text { www.karger.com/dpa }\end{array}$ \\
\hline
\end{tabular}

Duman et al.: Nevus-Associated versus de novo Melanoma: Do They Have Different Characteristics and Prognoses?

\begin{tabular}{|c|c|c|c|}
\hline & NAM & DNM & $\mathrm{p}$ \\
\hline Age, years & $49 \pm 15$ & $55 \pm 16$ & 0.057 \\
\hline Males/females & $18 / 10$ & $48 / 42$ & 0.423 \\
\hline Location & & & 0.005 \\
\hline Acral region & $3(10.7)$ & $28(31.1)$ & \\
\hline Trunk & $7(25)$ & $11(12.2)$ & \\
\hline Extremities & $14(50)$ & $22(24.4)$ & \\
\hline Head and neck region & $4(14.3)$ & $29(32.2)$ & \\
\hline Clinical stage & & & 0.505 \\
\hline 0 & $0(0)$ & $3(3.3)$ & \\
\hline $1 \mathrm{~A}$ & $6(21.4)$ & $13(14.4)$ & \\
\hline $1 \mathrm{~B}$ & $9(32.1)$ & $19(21.1)$ & \\
\hline $2 \mathrm{~A}$ & $3(10.7)$ & $8(8.9)$ & \\
\hline $2 \mathrm{~B}$ & $3(10.7)$ & $17(18.9)$ & \\
\hline $2 \mathrm{C}$ & $1(3.6)$ & $10(11.1)$ & \\
\hline 3 & $5(17.9)$ & $14(15.6)$ & \\
\hline 4 & $1(3.6)$ & $6(6.7)$ & \\
\hline Satellite metastasis ${ }^{1}$ & $2(7.1)$ & $3(3.3)$ & 0.591 \\
\hline In-transit metastasis ${ }^{1}$ & $1(3.6)$ & $3(3.3)$ & 1.000 \\
\hline Distant metastasis $^{1}$ & $3(10.7)$ & $26(28.9)$ & 0.089 \\
\hline
\end{tabular}

Figures are given as n (\%), unless otherwise specified.

${ }^{1}$ During a median follow-up of 3 years.
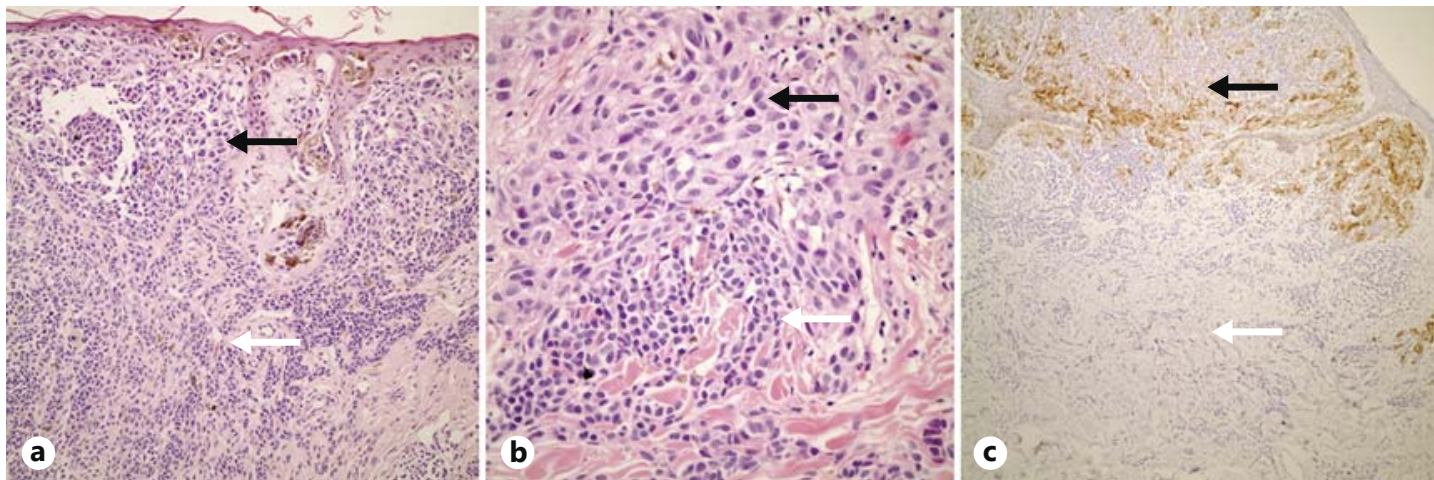

Fig. 1. Nevus-associated melanoma. a, b Melanoma cells on the upper side (black arrows) and nevus cells on the lower side (white arrows). c Melanoma cells stained positively with HMB-45 (black arrow), whereas nevus cells were HMB-45 negative (white arrow). HE. $\times 100$ (a), ×200 (b), ×80 (c).

\section{Results}

Among the 118 melanoma patients, 28 (23.7\%) had NAM (fig. 1), of whom 22 (78.6\%) reported a history of a preexisting nevus. Patient demographics are summarized in table 1. The distribution of gender was similar between the NAM and DNM patients. Although the mean age was lower in the NAM patients, the difference was not significant $(p=0.057)$. NAM was most commonly located on the extremities (50\%), followed by the trunk (25\%), whereas DNM was most commonly located in the head and neck region (32.2\%), followed by the acral region (31.1\%). In the NAM patients, 11 (39.3\%) had acquired a melanocytic nevus, $5(17.9 \%)$ had a dysplastic nevus, and $5(17.9 \%)$ had a congenital melanocytic nevus. In 7 (25\%) of the 
Table 2. Histopathological features in the NAM and DNM patients

\begin{tabular}{lccl}
\hline & NAM & DNM & $\mathrm{p}$ \\
\hline Median lesion size, cm & 1.2 & 1.5 & 0.185 \\
Ulceration & $8(28.6)$ & $43(47.8)$ & 0.116 \\
Median Breslow thickness, mm & 1.8 & 2.5 & 0.157 \\
Lymphovascular invasion & $2(7.1)$ & $12(13.3)$ & 0.514 \\
Perineural invasion & $2(7.1)$ & $4(4.4)$ & 0.627 \\
Lymphohistiocytic infiltration & & & \\
$\quad$ Absent & $10(35.7)$ & $30(33.3)$ & 0.830 \\
$\quad$ Non-brisk & $11(39.3)$ & $41(45.6)$ & \\
$\quad$ Brisk & $7(25)$ & $19(21.1)$ & \\
Regression & 2 & & \\
$\quad$ Partial regression $(<75 \%)$ & 0 & 10 & 0.752 \\
$\quad$ Complete regression $(\geq 75 \%)$ & $10(35.7)$ & $26(29.9)$ & \\
Mitotic index & $18(64.3)$ & $64(70.1)$ & \\
$\quad<1$ mm $^{-2}$ & & & \\
$\quad>1$ mm $^{-2}$ & & & \\
\hline
\end{tabular}

Figures are given as n (\%), unless otherwise specified.

NAM patients, the precursor nevus type could not be determined. Although the frequency was higher in the NAM patients, superficial spreading melanoma was the most common subtype both in the NAM (60.7\%) and DNM (35.6\%) patients. Histopathological parameters, including lesion diameter, Breslow thickness, Clark level, mitotic index, ulceration, lymphocytic infiltration and its type, regression and its type, and lymphovascular/perineural invasion, were similar in the NAM and DNM patients ( $\mathrm{p}>0.05$ ).

Sentinel lymph node examination was performed in 44 (49\%) of the DNM and in 10 (36\%) of the NAM patients. Among these patients, 10 (11\%) with DNM and 2 (7\%) with NAM had metastatic sentinel lymph node involvement $(\mathrm{p}=1.000)$. Regional lymph node dissection was performed in $25(27.8 \%)$ of the DNM patients and in $14(50 \%)$ of the NAM patients. Regional lymph node involvement was observed in $11(12 \%)$ of the DNM patients versus 4 $(14 \%)$ of the NAM patients $(p=0.544)$. Satellite metastasis was observed in $3(3.3 \%)$ DNM and $2(7.1 \%)$ NAM patients $(p=0.591)$, and in-transit metastasis was observed in $3(3.3 \%)$ DNM patients versus $1(3.6 \%)$ NAM patient $(\mathrm{p}=1.000)$.

The median duration of follow-up was similar in the NAM and DNM patients (NAM: 2.8 years; DNM: 3 years). The frequency of distant metastasis during follow-up did not differ significantly between the NAM and DNM patients (DNM: 28.9\%, $n=26$; NAM: $10.7 \%, n=3$; $\mathrm{p}=0.089$ ). A detailed comparison of the NAM and DNM patients is shown in tables 1 and 2 . Moreover, all analyses were repeated in cases in which NAM was defined based on patient anamnesis. Totally, 47 (39.8\%) of the NAM patients reported a history of a preexisting nevus. There were no significant differences in clinical, histopathological, and prognostic variables, except for the most common locations (extremities in the NAM patients: $38.3 \%$; head and neck region in the DNM patients: $36.6 \%$; $=0.005$ ), between the NAM and DNM patients.

\section{Discussion}

The precise frequency of NAM remains unknown. In fact, the criteria for defining melanoma as NAM is a contentious issue. Some studies considered the histopathologically proven presence of nevus cells as diagnostic for NAM, whereas others diagnosed NAM based on patient 
anamnesis [3-6]. However, the use of histological evidence of nevus cells to determine that a melanoma has arisen from a preexisting nevus, as in the present study, is associated with the following diagnostic difficulties: (1) since melanoma cells can destroy nevus cells as they grow, it is not always possible to observe the nevus cells in advanced cases of melanoma; (2) in rare cases, the coincidental coexistence of a nevus and an adjacent melanoma may lead to misdiagnosis, and (3) the histopathological misinterpretation of nevus cells as melanoma cells may lead to difficulty in evaluating Breslow thickness. Although patient history can be used to determine if a melanoma has arisen from a nevus and thus to diagnose NAM, it can also lead to misdiagnosis due the questionable accuracy of patient recall of a preexisting lesion.

Based on histopathological findings, approximately 30-33\% of melanomas develop from a precursor nevus $[3,7,8]$. Based on patient reports, the frequency of a precursor melanocytic nevus component ranges from 18 to $85 \%$ [4]. In the present study, $23.7 \%$ of the patients were diagnosed with NAM based on histopathological findings, versus $39.8 \%$ based on patient anamnesis. Another study that compared clinical and histopathological findings reported that $70 \%$ of patients with histopathological evidence of a precursor nevus reported a history of such, which is in agreement with the $22(78.6 \%)$ patients with histopathological findings of a precursor nevus in the present study who also reported a history of a preexisting nevus [9].

Earlier studies reported that most NAMs are located on the trunk [3-5, 8], whereas data on the most common locations of DNMs are inconsistent. Some studies reported that the head and neck region was the most common site of DNMs, whereas others reported that the trunk or the extremities was the most common location $[3,4,6]$. In the present study, most NAMs were located on the extremities and the trunk, and DNMs arose most commonly in the head and neck and the acral region. It also remains unclear if NAM and DNM have different prognoses. A clinicohistopathological study that included 557 patients (130 with NAM and 427 with DNM) reported that there were fewer cases of metastasis and/or death due to melanoma among the patients in the NAM group [9]. The researchers concluded that the presence of nevus cells in a melanoma specimen is associated with a better prognosis [9]. The study on patient anamnesis by Weatherhead et al. [3] reported that the median Breslow thickness of NAMs was greater than that of DNMs (1 vs. $0.7 \mathrm{~mm}$ ), and the researchers concluded that NAMs potentially have a poorer prognosis than DNMs. Although the difference between their NAM and DNM groups was not statistically significant independent of other factors, they suggested that a larger study might identify such a relationship [3]. In a recent study that included 1,190 melanoma patients (390 with NAM and 800 with DNM), Shitara et al. [7] reported that NAMs had a lower Breslow thickness compared to DNMs; however, they did not evaluate other prognostic factors such as survival, lymph node status, or development of distant metastasis between the two groups. The recent clinicohistopathological study by Lin et al. [8] included 850 melanoma patients (235 with NAM and 615 with DNM) and reported no association with sentinel lymph node status and no survival difference between NAMs and DNMs. In that study, the median Breslow thickness of DNMs was greater than that of NAMs (1.8 vs. $1.4 \mathrm{~mm}$ ); however, the difference was not significant. Similarly, in the present study, the Breslow thickness in DNMs was greater than that in NAMs (2.5 vs. $1.8 \mathrm{~mm}$ ), but the difference was not significant. Furthermore, other prognostic factors, including the development of sentinel/regional lymph node or distant metastasis, did not significantly differ between the NAM and DNM patients. Based on the present findings, we think that NAMs and DNMs have similar prognoses.

The present study has some limitations: (1) the retrospective design; (2) the small number of NAM patients; (3) histopathological difficulties, as mentioned above, and (4) a short duration of follow-up. Although there was no significant difference in the frequency of regional/distant metastasis between the NAM and DNM patients during a median follow-up of 3 years, this finding must be confirmed by studies with longer follow-ups. 


\section{Conclusions}

In conclusion, in the present study, NAM was diagnosed based on histopathological evidence of a precursor nevus. Most NAMs were located on the extremities and trunk, whereas most DNMs were located in the head and neck region as well as in the acral region. There were no significant differences in the other clinical, histopathological, or prognostic parameters between the NAM and DNM patients. Furthermore, the present findings remained consistent when NAM was diagnosed based on patient anamnesis.

\section{Disclosure Statement}

The authors have no conflicts of interest to declare.

\section{References}

1 Mangas C, Paradelo C, Puig S, Gallardo F, Marcoval J, Azon A, Bartralot R, Bel S, Bigatà X, Curcó N, Dalmau J, del Pozo LJ, Ferrándiz C, Formigón M, González A, Just M, Llambrich A, Llistosella E, Malvehy J, Martí RM, Nogués ME, Pedragosa R, Rocamora V, Sàbat M, Salleras M: Initial evaluation, diagnosis, staging, treatment, and followup of patients with primary cutaneous malignant melanoma. Consensus statement of the Network of Catalan and Balearic Melanoma Centers. Actas Dermosifiliogr 2010;101:129-142.

2 Bastian BC, Lazar A: Melanoma; in Calonje E, Brenn T, Lazar A, Mckee PH (eds): Mckee's Pathology of the Skin. China, Elsevier-Saunders, 2012, vol 2, pp 1221-1267.

3 Weatherhead SC, Haniffa M, Lawrence CM: Melanomas arising from naevi and de novo melanomas - does origin matter? Br J Dermatol 2007;156:72-76.

-4 Garcia-Cruz A, Flórez A, de la Torre-Fraga C, Cruces Prado M: Observational cross-sectional study comparing Breslow thickness of melanoma arising from naevi and melanoma de novo. Br J Dermatol 2009;161:700-702.

-5 Bevona C, Goggins W, Quinn T, Fullerton J, Tsao H: Cutaneous melanomas associated with nevi. Arch Dermatol 2003;139:1620-1624.

6 Harley S, Walsh N: A new look at nevus-associated melanomas. Am J Dermatopathol 1996;18:137-141.

7 Shitara D, Nascimento MM, Puig S, Yamada S, Enokihara MM, Michalany N, Bagatin E: Nevus-associated melanomas: clinicopathologic features. Am J Clin Pathol 2014;142:485-491.

$>8$ Lin WM, Luo S, Muzikansky A, Lobo AZ, Tanabe KK, Sober AJ, Cosimi AB, Tsao H, Duncan LM: Outcome of patients with de novo versus nevus-associated melanoma. J Am Acad Dermatol 2015;72:54-58.

-9 Friedman RJ, Rigel DS, Kopf AW, Lieblich L, Lew R, Harris MN, Roses DF, Gumport SL, Ragaz A, Waldo E, Levine J, Levenstein M, Koenig R, Bart RS, Trau H: Favorable prognosis for malignant melanomas associated with acquired melanocytic nevi. Arch Dermatol 1983;119:455-462. 